\title{
Exercícios físicos em pacientes internados em Unidade de Terapia Intensiva: novas perspectivas
}

\author{
Rodrigo Daminello Raimundo \\ Faculdade de Saúde Pública da Universidade de São Paulo (USP) - São Paulo (SP), Brasil \\ E-mail: rodaminello@yahoo.com.br
}

DOI: http://dx.doi.org/10.7322/abcshs.v40i1.694

O uso de exercícios físicos em pacientes internados em unidade de terapia intensiva (UTI) vem aumentando nos hospitais brasileiros. A fisioterapia respiratória hospitalar sempre deu ênfase para a mobilização de pacientes internados; porém, nos últimos anos, as pesquisas científicas têm buscado evidências de melhora em marcadores séricos, parâmetros hemodinâmicos e, principalmente, em diminuição no tempo de internação ${ }^{1}$.

Dentro deste contexto, neste fascículo da ABCS Health Sciences, o estudo de Costa et al. ${ }^{2}$ mostra como exercícios físicos aplicados de forma protocolada podem ser eficazes na redução do tempo de internação de pacientes hospitalizados. Este elegante estudo randomizou 26 pacientes internados em dois grupos, sendo que o grupo controle realizou fisioterapia tradicional e outro grupo foi treinado com cicloergômetro e exercícios neuromusculares. Os resultados mostraram que o grupo com exercícios neuromusculares e cicloergômetro reduziu o tempo de internação e, além disso, teve um melhor controle da pressão arterial (PA) quando comparado com um programa de fisioterapia tradicional.

Os efeitos deletérios do repouso são estudados há anos por pesquisadores do mundo todo, a história da pesquisa sobre este tema está enlaçada com o aparecimento de estudos que mostravam que a inatividade e o descondicionamento físico de pacientes em leitos de hospital eram extremamente prejudiciais em vários sistemas do corpo humano, como no sistema musculo-esquelético (hipotrofismo, osteoporose, deformidades articulares), no sistema respiratório (diminuição da força de musculatura respiratória e predisposição para infecções pulmonares), no sistema cardiovascular (alteração na volemia, alterações na pressão arterial sistêmica e predisposição para trombose venosa profunda, aumentando o risco para embolia pulmonar), além de alterações renais e gastrointestinais ${ }^{1}$. Há interessantes relatos na literatura, como a pesquisa de Herridge et al. ${ }^{3}$, que relacionam a fraqueza muscular adquirida nas internações hospitalares com uma das razões de desemprego destes pacientes pela alta taxa de fadiga e fraqueza, mesmo após um ano de alta hospitalar.

Em 2008, Gosselink et al. ${ }^{4}$ publicaram as recomendações da European Respiratory Society (ERS) e da European Society of Intensive Care Medicine (ESICM) de fisioterapia para pacientes críticos. As evidências foram limitadas a níveis de recomendações "C" (evidências de ensaios não controlados ou não randomizados, ou a partir de estudos observacionais) e D (opinião de especialistas). Dentre estas evidências destacaram-se a avaliação e a monitorização adequada das funções vitais para garantir um nível seguro de intervenção. Visto isso, neste fascículo da ABCS Health Sciences, destaca-se uma outra pesquisa feita por Baltieri et al. ${ }^{5}$, que avaliou a força muscular respiratória (manovacuometria), a função pulmonar (espirometria) e a capacidade funcional de pacientes (Teste de caminhada dos 6 minutos - TC6M) no pré-operatório de ressecção pulmonar por neoplasia. Foram avaliados os valores de pressão expiratória máxima, capacidade vital forçada, volume expiratório forçado no primeiro segundo (VEF1), razão VEF1/CVF e distância percorrida pelo TC6M de 59 pacientes. A pesquisa mostrou prejuízos na força muscular expiratória, na função pulmonar e na capacidade funcional neste perfil de pacientes.

Dentro das recomendações da ERS ${ }^{4}$, existe um destaque para mobilização e treinamento muscular precoce e o posicionamento adequado do paciente no leito, com alongamentos musculares para preservar a mobilidade articular. Um dos grandes problemas no atendimento motor de pacientes críticos é a dificuldade de fazer diagnósticos e, por sua vez, traçar tratamentos adequados para doenças como miopatias aguda, fraqueza muscular e polineuropatias ${ }^{1}$. Várias escalas têm sido usadas para a tentativa de avaliar o grau de fraqueza muscular de pacientes internados em UTI, umas das escalas mais usadas é a da Medical Research Council (MRC), que avalia grupos musculares específicos - valores abaixo de 48 pontos, nesta escala, denotam maior rigor no tratamento da fraqueza muscular de pacientes graves. 
Atualmente, os exercícios motores frequentemente usados em hospitais estão baseados em exercícios de membros superiores e inferiores; treinamento funcional; mudança e transferências para fora do leito; deambulação e exercícios respiratórios. Dentro das novas perspectivas para melhor conduta, destacam-se o uso cada vez mais precoce de pranchas ortostáticas, cicloergômetros de membros superiores e inferiores, estimulação elétrica neuromuscular, ortostatismo e deambulação ${ }^{1}$. Fatores como idade avançada, diabetes mellitus, anormalidades metabólicas, uso prolongado de medicações de corticoides e sedativos, elevado tempo de permanência na UTI e de ventilação mecânica (VM) podem contribuir para a ocorrência de neuropatias ${ }^{6}$.

Duas intervenções merecem destaque no atendimento de pacientes internados em hospitais contra a redução de massa muscular: a eletroestimulação e o uso de cicloergômetros. Estudos têm mostrado que a eletroestimulação pode ser um recurso coadjuvante interessante para o aumento de massa muscular de pacientes que já estão acometidos com polineuropatias, porém não têm mostrado eficácia para a prevenção destas patologias. Concomitantemente, os exercícios em cicloergômetro tanto com os membros superiores como inferiores indicam um aumento da capacidade funcional e da força muscular desta população ${ }^{6-8}$.

Apesar de avanços e de cada vez mais aparecerem trabalhos científicos sobre o assunto, a mobilização precoce e o exercício físico de pacientes internados apresentam dificuldades de implementação em hospitais. Vários trabalhos ${ }^{8-11}$ mostram que a implementação de protocolos é víavel e segura mas necessita-se de uma mudança de paradigma de toda a equipe multiprofissional para uma melhora na atenção de pacientes hospitalizados.

\section{REFERÊNCIAS}

1. Raimundo RD. Fisioterapia cardiovascular e metabólica. São Paulo: Atheneu; 2013.

2. Costa MFL, Patto J, Gomes VA, Prazeres LS, Santos ACN, Almeida MCG, et al. Efeitos dos exercícios funcionais e neuromusculares no tempo de internação e controle pressórico de pacientes hospitalizados. ABCS Health Sci. 2015;40(1):33-7. http://dx.doi.org/10.7322/abcshs.v40i1.701

3. Herridge MS, Cheung AM, Tansey CM, Matte-Martyn A, DiazGranados N, Al-Saidi F, et al. One-year outcomes in survivors of the acute respiratory distress syndrome. $N$ Engl $\mathrm{J}$ Med. 2003;348(8):683-93.

http://dx.doi.org/10.1056/NEJMoa022450

4. Gosselink R, Bott J, Johnson M, Dean E, Nava S, Norrenberg $\mathrm{M}$, et al. Physiotherapy for adult patients with critical illness: recommendations of the European Respiratory Society and European Society of Intensive Care Medicine Task Force on Physiotherapy for Critically III Patients. Intensive Care Med. 2008;34(7):1188-99. http://dx.doi.org/10.1007/s00134-008-1026-7

5. Baltieri L, Passos ASM, GalhardoFDM, Roceto LS, Toro IFC. Avaliação pré-operatória da força muscular respiratória, da função pulmonar e da capacidade funcional de pacientes submetidos à ressecção pulmonar. ABCS Health Sci. 2015;40(1):22-7. http://dx.doi.org/10.7322/abcshs.v40i1.699
6. Pinheiro AR, Christofoletti G. Motor physical therapy in hospitalized patients in an intensive care unit: a systematic review. Rev Bras Ter Intensiva. 2012;24(2):188-96

http://dx.doi.org/10.1590/S0103-507X2012000200016

7. Gruther W, Kainberger F, Fialka-Moser V, Paternostro-Sluga T, Quittan $\mathrm{M}$, Spiss $\mathrm{C}$, et al. Effects of neuromuscular electrical stimulation on muscle Layer thickness of knee extensor muscles in intensive care unit patients: a pilot study. J Rehabil Med. 2010;42(6):593-7. http://dx.doi.org/10.2340/16501977-0564

8. Burtin C, Clerckx B, Robbeets C, Ferdinande P, Langer D, Troosters $\mathrm{T}$, et al. Early exercise in critically ill patients enhances short-term functional recovery. Crit Care Med. 2009;37(9):2499-505. http://dx.doi.org/10.1097/CCM.0b013e3181a38937

9. Roberts M, Johnson LA, Lalonde TL. Early mobility in the intensive care unit: standard equipment vs a mobility platform. Am J Crit Care. 2014;23(6):451-7. http://dx.doi.org/10.4037/ajcc2014878

10. Brownback CA, Fletcher P, Pierce LNB, Klaus S. Early mobility activities during continuous renal replacement therapy. Am J Crit Care. 2014;23(4):348-51. http://dx.doi.org/10.4037/ajcc2014889

11. Blot S, Afonso E, Labeau S. Recent advances in multidisciplinary critical care. Am J Crit Care. 2015;24(1):75-86. http://dx.doi.org/10.4037/ajcc2015321. 